\title{
ABHANDLUNGEN DER DEUTSCHEN AKADEMIE DER WISSENSCHAFTEN ZU BERLIN
}

\author{
Klasse für Mathematik, Physik und Technik \\ Jahrgang 1960 Nr. 7
}

\section{REAKTIONSKINETIK VON ELEKTRONENPROZESSEN IN FESTKÖRPERN}

\author{
Tagung \\ der Deutschen Akademie der Wissenschaften zu Berlin \\ Physikalisch-Technisches Institut \\ - Bereich Elektrischer Durchschlag - \\ vom 11. - 13. April 1960
}

Herausgegeben von KARL WOLFGANG BÖER

Mit 126 Abbildungen und 12 Tabellen

AKADE M IE - VERLAG - BERLIN

1960 
Vorgelogt von Hm. ROMPE in der Klassensitzung vom 11. Februar 1960

Zum Druck genehmigt am gleichen Tage, ausgegeben am 30. Juni 1960

Erachionen im Akademio-Vorlag GmbH, Bertin W 1, Leipriger Str. 3-4 Lizens-Nr. $202 \cdot 100 / 766 / 60$

Alle Rèahte vorbahalien. Copyright 1960 by Akademio-Verlag GmbH Gesamtherotellung: VEB Druckerei ,Thomas Montrer" Bad Langencelsa Bestellonmmer: 3001/60/1/7

Printed in Gormany

Is 18 B 6/7 